\title{
A Yabloesque paradox in epistemic game theory
}

\author{
Can Başkent ${ }^{1}$
}

Received: 12 April 2016 / Accepted: 22 September 2016/ Published online: 1 October 2016

C The Author(s) 2016. This article is published with open access at Springerlink.com

\begin{abstract}
The Brandenburger-Keisler paradox is a self-referential paradox in epistemic game theory which can be viewed as a two-person version of Russell's Paradox. Yablo's Paradox, according to its author, is a non-self referential paradox, which created a significant impact. This paper gives a Yabloesque, non-self-referential paradox for infinitary players within the context of epistemic game theory. The new paradox advances both the Brandenburger-Keisler and Yablo results. Additionally, the paper constructs a paraconsistent model satisfying the paradoxical statement.
\end{abstract}

Keywords Yablo's Paradox · The Brandenburger-Keisler paradox · Epistemic game theory $\cdot$ Paraconsistent logic

\section{Introduction}

In this paper, we present a direct application of Yablo's Paradox to a problem in epistemic game theory using the language of propositional bimodal logic. Yablo's paradox, according to its author, is a non-self-referential paradox and it has created a significant attention in the literature, especially from the perspectives of truth theory and fixed-point logics. Here, we extend the discussion to theory of games and give a Yabloesque, non-self-referential paradox within the context of epistemic game theory.

We have two specific goals in this work. The first is to apply Yablo's argument to a field which can provide some further insight for the discussions regarding the self-referentiality of Yablo's paradox. As we discuss later on, it is not entirely obvious

\footnotetext{
$凶$ Can Başkent

can@canbaskent.net

http://www.canbaskent.net/logic

1 Department of Computer Science, University of Bath, Bath, UK
} 
whether Yablo's paradox is genuinely self-referential. Therefore, presenting different approaches to the paradox within different formal frameworks is a reasonable direction to pursue. Our second goal is to give a non-classical solution, that is a paraconsistent model, for the game theoretical Yabloesque paradox. Therefore, this paper suggests a newly formulated paradox in epistemic game theory and a paraconsistent model for it. The two goals of the current work are closely connected both logically and game theoretically. The logical connection is clear. We give a direct application of paraconsistency and address the challenge of developing paraconsistent models with immediate applications. Therefore, the current work contributes to the discussions on the ontological possibilities of paraconsistency. Game theoretical connection, on the other hand, suggests the possibility of developing real-life game models where such paradoxical cases may arise. This connection offers additional tools and techniques for game theory. From a broader perspective, therefore, our programmatic goal for studying game theoretical paradoxes is to take a first step towards understanding paraconsistent games-games that can have non-trivial inconsistent models where agents may possess inconsistent knowledge or may make inconsistent moves.

The choice of epistemic game theory is not arbitrary. Some years ago, a selfreferential paradox was identified in epistemic game theory by Brandenburger and Keisler (2006). The game theoretical contribution of the current paper is complementary to the work of Brandenburger and Keisler where we now suggest a non-self-referential paradox in epistemic game theory.

In what follows, we start with reviewing first the Brandenburger-Keisler paradox, then Yablo's paradox, followed by a brief overview of the literature.

\subsection{The Brandenburg-Keisler paradox}

The Brandenburg-Keisler paradox ('BK paradox', henceforth) is a two-person selfreferential paradox in epistemic game theory (Brandenburger and Keisler 2006). The paradox arises when the following sentence is considered for two players Ann and Bob:

Ann believes that Bob assumes that Ann believes that Bob's assumption is wrong.

and the question if "Ann believes that Bob's assumption is wrong", where Bob's assumption is the sentence that "Ann believes that Bob's assumption is wrong" is considered.

If the answer to the above question is a "yes", then Ann does believe that Bob's assumption is wrong, which means that she believes that the statement "Bob's assumption is wrong" is wrong. Therefore, Ann believes that Bob's assumption is correct. But, initially she believed that this assumption was wrong. This creates a contradiction. On the other hand, if the answer is "no", then she does not believe that Bob's assumption is wrong, which means that Ann believes that Bob's assumption is correct. However, this contradicts the assumption that "Ann believes that Bob's assumption is wrong". This is a contradiction, too. Both possible answers to the question create a contradiction. Thus, we obtain a paradox. The BK paradox, as the above reasoning demonstrates, can be seen as a two-person liar's paradox. The paradox is indeed self-referential, 
expressible with a fixed-point operator (Abramsky and Zvesper 2015). The paradox shows that players' beliefs and assumptions cannot completely be modeled, that is "not every description of belief can be represented" with belief structures (Brandenburger and Keisler 2006).

Following, there have been several attempts to represent the BK paradox in different frameworks including a hybrid logical (Pacuit 2007), an algebraic and categorical theoretical (Abramsky and Zvesper 2015), and a paraconsistent and non-well-founded set theoretical (Başkent 2015) approaches. Even if the paradox was given for two players, it can also be extended to countably-many players (Abramsky and Zvesper 2015). Furthermore, similar to various non-classical logical solutions suggested for liar paradoxes, there has been suggested a variety of non-classical models which satisfy the BK paradox (Başkent 2015).

The BK paradox is formalized using a bimodal language and relational models. We briefly review the logical framework on which we shall build our new paradox. The model $M=\left(U^{a}, U^{b}, R^{a b}, R^{b a}, V\right)$ is called a belief model where $U^{a}$ and $U^{b}$ are the set of possible worlds (sometimes called "type spaces") for players Ann and Bob, respectively; $R^{a b} \subseteq U^{a} \times U^{b}$ and $R^{b a} \subseteq U^{b} \times U^{a}$, and $V$ is a valuation function defined in the standard way. The expression $R^{a b}(x, y)$ represents that in state $x$, Ann believes that the state $y$ is possible for Bob, and vice versa for $R^{b a}(y, x)$. We put $R^{a b}(x)=\left\{y: R^{a b}(x, y)\right\}$, and similarly for $R^{b a}(y)$. At a state $x$, we say Ann believes $P \subseteq U^{b}$ if $R^{a b}(x) \subseteq P$. It is important to note that the belief relation $R^{i j}$ is given for $i \neq j$ disallowing self-referentiality for beliefs. In other words, the self-referentiality we consider is not directly about the beliefs of agents about themselves.

The formal language used to express the BK paradox is a propositional bimodal language with the modalities $\square^{i j}$ and $\varnothing^{i j}$ which stand for player $i$ 's belief and assumption about player $j$ respectively. The semantics for the interactive belief structures is given as follows, where the Boolean cases are omitted as they are standard.

$$
\begin{array}{ll}
x \models \square^{a b} \varphi & \text { iff } \quad \forall y \in U^{b} \cdot R^{a b}(x, y) \text { implies } y \models \varphi \\
x \models \nabla^{a b} \varphi & \text { iff } \quad \forall y \in U^{b} \cdot R^{a b}(x, y) \text { iff } y \models \varphi
\end{array}
$$

The assumption modality is worth revisiting. It can be argued that the assumption modality, as it stands, does not precisely express what is usually meant by an assumption in formal sciences and game theoretical reasoning. Rather, it suggests a form of belief, a strongest belief perhaps. ${ }^{1}$ Reading the assumption modality as the "strongest belief" does not affect our results in this work. Nevertheless, for pragmatic reasons, we will adhere to the original terminology and call the relevant modality as the assumption modality.

Based on this framework, it was shown that not every configuration of beliefs and assumptions are representable in belief models, such as the BK paradox (Branden-

\footnotetext{
1 The original formulation of the BK paradox uses the term assumption to describe strongest beliefs. These are the beliefs that perfectly match with the set of accessible states from the current one. Therefore, it can be suggested that the term assumption is slightly misleading. Even if that is not our concern for the purposes of the current work, there is some truth in this argument. In order not to make terminology more complicated, we chose to stick to the current naming conventions for the modalities. I am thankful to Eric Pacuit for drawing my attention to this point.
} 
burger and Keisler 2006). We refer the reader to the original work for the technical details of the impossibility result.

\subsection{Yablo's paradox and its impact}

Yablo's Paradox, according to its author, is a non-self referential paradox (Yablo 1985, 1993). The paradox is given by considering the following sequence of sentences.

$$
\begin{aligned}
& S_{1}: \forall k>1, S_{k} \text { is untrue, } \\
& S_{2}: \forall k>2, S_{k} \text { is untrue, } \\
& S_{3}: \forall k>3, S_{k} \text { is untrue, }
\end{aligned}
$$

By using reductio, Yablo shows that every sentence $S_{n}$ is untrue. Then, "the sentences subsequent [his emphasis] to any given $S_{n}$ are all untrue, whence $S_{n}$ is true after all!", which is a contradiction (Yablo 1993). Here, the infinitary nature of the paradox is essential as each finite set of $S_{n}$ is satisfiable.

The scheme of this paradox is not new. To the best of our knowledge, the first analysis of a paradox with the same pattern was suggested by Yuting (1953).

The paradox received significant attention in the literature, including a truth theoretical debate over whether it is genuinely a self-referential paradox. However, it is not our focus here to discuss the truth-theoretical conditions of the paradox. Nevertheless, we hope that the game theoretical analysis will have some potential to shed light on the truth theoretical debate. Notwithstanding, in what follows we briefly review some of the discussions on the paradox.

Ketland showed that the paradox is $\omega$-inconsistent and gave a general scheme for Yablo-like sentences with $\omega$-inconsistency (Ketland 2005). Furthermore, Barrio showed that Yablo's Paradox in first-order arithmetic has a model and not inconsistent, but it is $\omega$-inconsistent (Barrio 2010). It is easy to see how. Since every finite set of $S_{n}$ sentences is satisfiable, then, by compactness there exists a model for the Yablo sentences. By $\omega$-inconsistency, it can be argued that the model we are looking for is a non-standard model of arithmetic. We will discuss categoricity in due time.

The relation between liar-like circular paradoxes and Yablo's paradox received significant attention as the aforementioned relation is far from being trivial. As Hardy puts it "Is Yablo's paradox Liar-like? In some ways yes, and in other ways no" (Hardy 1995). Priest, however, offers another analysis regarding the infinitary language that it requires, and suggests a reading of the paradox that does indeed involve circularity (Priest 1997). Sorensen disagrees and point out the hierarchical view of Tarskian truth theory arguing that Yablo's paradox in effect "exploit[s] an alternative pattern of semantic dependency" (Sorensen 1998). Beall extends the discussion on the selfreferentiality of the paradox and argues on the non-circularity of the paradox even if he thinks that Sorensen's arguments do not address Priest's point accurately (Beall 2001).

There have been a variety of applications of Yablo's paradox in logic. Goldstein presents a set theoretical Yabloesque paradox for class membership (Goldstein 1994). A further interesting application of Yablo argument is Goldstein's investigation of 
the concept by using Fibonacci sequences to illustrate the under-specification of the truth conditions of he paradox (Goldstein 2006) Leitgeb, on the other hand, suggests a Yabloesque paradox for non-well-founded definitions which underlines the set theoretical limitations of the logical toolbox (Leitgeb 2005). Picollo discusses the paradox from a second-order logical perspective generalizing the $\omega$-inconsistency results (Picollo 2013). Another line of generalization is concerned with the non-wellfoundedness of Yablo sentences and relates it to similar discussions in foundational set theory (Bernardi 2001). Non-well-founded Yablo chains form a topological space which can be viewed as the underlying idea of Bernardi's topological approach to the paradox (Bernardi 2009). Cook, on the other hand, considers Yablo's paradox and gives Curry-like versions of the paradox (Cook 2009). Beall, moreover, gives a nonmodal Yabloesque curry paradox which initially triggers Cook's approach which we have mentioned (Beall 1999).

A recent monograph by Cook discusses the paradox at length providing both further philosophical insight and a detailed overview of the literature (Cook 2014). Cook considers two broad issues. The first is understanding the paradox by examining "whether the Yablo paradox (or some modification of it) is genuinely non-circular, or whether the non-circularity is merely apparent" [Cook (2014), §1]. The second is to study if it is possible to "apply the general Yabloesque pattern to other paradoxes, replacing each circular paradox with a non-circular Yabloesque analogue, thereby eliminating the need to make use of circular constructions altogether" which he calls as "the Generalizibility Question" [ibid]. Our study, therefore, provides a direct response to the "Generalizibilty Question" by presenting a game theoretical paradox with the Yabloesque pattern.

In this paper, we reformulate Yablo's paradox in an epistemic game theoretical context complementing and extending the BK paradox. Our semantic approach directly relates the discussion to both truth theories and epistemic game theory, thus helps unravel the complications that paradoxes create in both fields. Moreover, as the standard language of epistemic game theory is modal, our analysis can be viewed as a bimodal extension of the Yablo paradox.

In the following, we first introduce the argument, followed by its formalization. Before proving the impossibility result, we discuss an instantiation of the paradoxical sentence for illustrative purposes. Consequently, we present a brief discussion on how the new paradox relates to major issues in logic. Finally, we propose a paraconsistent model for the paradoxical sentence, observing under which conditions the new paradox can be satisfied.

\section{A non-self referential epistemic game theoretical paradox}

\subsection{The argument}

Let us consider the following sequence of assumptions where numerals represent game theoretical players. 
$A_{1}: 1$ believes that $\forall k>1, k$ 's assumption $A_{l}$ about $\forall l>k$ is untrue,

$A_{2}: 2$ believes that $\forall k>2, k$ 's assumption $A_{l}$ about $\forall l>k$ is untrue,

$A_{3}: 3$ believes that $\forall k>3, k$ 's assumption $A_{l}$ about $\forall l>k$ is untrue,

The above set of statements is slightly complicated (especially, compared to the Yablo sentences). So, first we present an informal argument which shows why and how the above set of sentences is paradoxical.

What does the above statement mean in an epistemic game theoretical framework? First of all, it is a game with $\omega$-many players as the statement is $\omega$-inconsistent. It is not difficult to imagine a situation that can illustrate the situation game theoretically. Let us give an example similar to Sorensen (1998). While discussing Yablo's paradox, Sorensen gives the well-known quеие example, which is the Yablo argument in a dual form. He considers an infinite queue of students where each students think "Some of the students behind me are now thinking an untruth" [ibid]. Sorensen shows that this is a paradoxical situation.

Now, similarly, imagine a queue of players, where players are conveniently named after numerals, holding beliefs about each player behind them, but not about themselves. In this case, each player $i$ believes that each player $k>i$ behind them has an assumption about each other player $l>k$ behind them, and $i$ believes that each $k$ 's assumption is false. This statement is perfectly perceivable for games, and involves a specific configuration of players' beliefs and assumptions, which is expressible in the language. However, as we shall show, similar to Yablo's paradox and the BK paradox, this configuration of beliefs is impossible. Moreover, the paradox is more complex than Sorensen's.

It is so because, for example, Student 7 is certainly subject to some students' beliefs in more than one way, and the relation between different students with assumptions about Student 7 is strong, perhaps too strong. For instance, it is believed by Student 3 what Student 5 assumes about Student 7 is untrue. Moreover, it is also the case that Student 4 believes what Student 6 assumes about Student 7 is untrue. This is where the assumption modality makes a formal difference and associates the assumptions of Student 5 and 6 with each other. The assumption modality precisely identifies the set of possible worlds at which the assumption is true. By doing so, the assumptions of Student 5 and Student 6 about Student 7 are satisfied at the very same set of possible worlds to which they both have access. Therefore, since the same sentence is assumed by different students in the queue, the same set of possible worlds are forced to be available to all these students by the definition of the assumption modality. This setup introduces an additional level of formal and semantical complexity to the paradox which cannot be eliminated by replacing the problematic part with a predicate (such as UNTRUE). The reason stems from the interactive nature of the paradox and how different students assuming the same untruth are identified using the semantics of the assumption modality. As we shall discuss later on, the assumption modality is indispensable in this version 
of the paradox as the paradoxical situation does not appear using only the belief modality. $^{2}$

Now, we can observe why the set of sentences given at the beginning of this section is not self-referential. For each $n \in \omega$, the predicates in $A_{n}$ ranges over $m>n$, but not over $n$. The sentence $A_{n}$ does not contain any predicate about player $n .^{3}$

Let us start with an informal semantic argument. Now, for a contradiction, assume $A_{n}$ is true for some $n$. Therefore, player $n$ believes that $\forall k>n, k$ 's assumption is untrue. In particular, player $n+1$ 's assumption is untrue. In other words, $n+1$ 's assumption

$$
A_{n+1}: n+1 \text { believes that } \forall k>n+1, k \text { 's assumption } A_{l} \text { about } \forall l>k \text { is untrue. }
$$

Therefore, $n+1$ believes that for some $k^{\prime}>n+1$, what $k^{\prime}$ assumes about some $l^{\prime}>k^{\prime}$ is true. But, this combination of players $k^{\prime}$ and $l^{\prime}$, both of which are bigger than $n+1$, thus $n$, is accessible from $n$ by means of the belief-assumption modalities. We assumed $A_{n}$ is true, which entails that what $k^{\prime}$ assumes about some $l^{\prime}>k^{\prime}$ is untrue. Contradiction. The choice of $n$ was arbitrary, so each $A_{n}$ in the sequence is untrue.

However, if each $A_{n}$ is untrue, they can be assumed untrue in some model. But, if for all $n, n$ 's assumption is untrue, then $A_{1}$ is indeed true. Yet, we just argued that each $A_{n}$ is untrue. Contradiction. Thus, the paradox has no model.

Some remarks about the above reasoning are in order. First, the initial inconsistency occurs since players whose numerals are large enough can be accessed by the modalities both from the sentence which was initially assumed true and from the sentence that is assumed to be untrue (by the initially assumed true sentence). And this creates a contradiction, similar to Yablo's original arguments. This fact relates the paradox to non-well-foundedness as observed by Yuting (1953). Moreover, it is important to note that the informal argument above requires non-finite resources to prove the contradiction. Our semantic approach, however, shows that the infinitary set of sentences given above has no classical model, reasoning at the level of metalanguage. ${ }^{4}$ In what follows, we shall set aside the proof theoretical and computational concerns and follow a semantical approach to argue that there is no classical model for our paradox.

Next, more importantly, in game theory, players are entitled to have their own beliefs and two players may coherently have contradictory beliefs. However, notice that the above contradiction is a contradiction about the truth of a statement, not about the belief of some truth.

\footnotetext{
2 In Sorensen (1998), Sorensen's queue example uses the modality think in an informal way. It is not clear if it can be formalized or if it can have a complete semantics in a modal or first-order framework. From a formalist perspective, the current paper supplements Sorensen's approach by introducing additional formal elements to the discussion. The beliefs and assumptions discussed in this paper are not just doxastic elements but also well-defined modal operators with clear semantics and proof theory.

3 As argued by Priest, the sentences contain quantification $\forall m>n$ which may be viewed self-referential (Priest 1997).

${ }^{4}$ I am grateful to the referee for bringing this matter to my attention.
} 


\subsection{Formal matters}

Let us be more formal now.

The Yabloesque Brandenburger-Keisler paradox ('YBK Paradox', henceforth) requires $\omega$-many players $i \in I$. The syntax of this language is given in the BackusNaur form as follows for a set of propositional variables $\mathbf{P}$ :

$$
\varphi:=p|\neg \varphi| \varphi \wedge \varphi\left|\square^{i j} \varphi\right| \varnothing^{i j} \varphi
$$

where $p \in \mathbf{P}$ and $i \neq j$ for $i, j \in I$ with $|I|=\omega$. The disjunction and implication are taken as abbreviations in the standard way.

The extended belief model is a tuple $M=\left(\left\{U^{i}\right\}_{i \in I},\left\{R^{i j}\right\}_{i \neq j \in I}, V\right)$ where $R^{i j} \subseteq$ $U^{i} \times U^{j}$ and $V$ is a valuation function defined in the standard way. As before, the expression $R^{i j}(x, y)$ represents that in state $x$, the player $i$ believes that the state $y$ is possible for player $j$. As before, we prevent (a trivial form of) self-reference by disallowing players having beliefs about themselves. This is indeed one of the points of paraconsistent approaches to the classical BK paradox (Başkent 2015).

The semantics for the modal operators is given as follows in a similar way.

$$
\begin{array}{ll}
x \models \square^{i j} \varphi & \text { iff } \quad \forall y \in U^{j} \cdot R^{i j}(x, y) \text { implies } y \models \varphi \\
x \models \nabla^{i j} \varphi & \text { iff } \quad \forall y \in U^{j} \cdot R^{i j}(x, y) \text { iff } y \models \varphi
\end{array}
$$

For a formula $\varphi$ in the language given, $|\varphi|$ denotes the set of states which satisfies $\varphi$. Formally, $|\varphi|=\left\{w \in \cup_{i \in I} U^{i}: w \models \varphi\right\}$. Additionally, $|\varphi|_{i}=\left\{w \in U^{i}: w \models \varphi\right\}$. We call $|\varphi|^{M}$ the extension of $\varphi$ in model $M$. We omit the superscript when it is obvious.

Now, we can formalize the YBK paradox in extended belief models by considering the following sequence of assumptions $A_{i}$ :

$$
\begin{aligned}
& A_{1}:=\bigwedge_{k>1} \square^{1 k}\left\{\bigwedge_{l>k} \nabla^{k l} \neg A_{l}\right\} \\
& A_{2}:=\bigwedge_{k>2} \square^{2 k}\left\{\bigwedge_{l>k} \nabla^{k l} \neg A_{l}\right\} \\
& A_{3}:=\bigwedge_{k>3} \square^{3 k}\left\{\bigwedge_{l>k} \nabla^{k l} \neg A_{l}\right\} \\
& \text { : }
\end{aligned}
$$

In the above set of sentences, each assumption $A_{i}$ is associated with player $i$ and numerals identify the players. By using semantical methods, we will now show that the above configuration of beliefs is (classically) impossible - that is there is no standard model for the infinite set of sentences given in Statement 1 above.

Here, $A_{1}$ is the assumption that the player 1 believes that all the other players $k>1$ believe that $k$ 's assumption $A_{l}$ about player $l>k$ is untrue. Similarly, for all other players. 
Following the ideas suggested in the informal proof we have given earlier, we assume that $A_{n}$ is true for some $n$ at some state $w \in U^{n}$. That is $w \models A_{n}$. Explicitly, we have

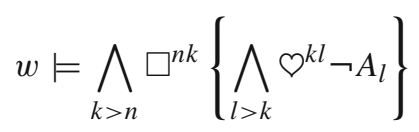

where $\neg A_{n}$ is given as follows:

$$
\neg A_{n}=\bigvee_{k>n} \diamond^{n k}\left\{\bigvee_{l>k} \neg \diamond^{k l} \neg A_{l}\right\}
$$

For clarity, let us specify the semantics of $\neg \bigodot^{i j} \neg \varphi$ which appears in $\neg A_{n}$ :

$$
x \models \neg \bigotimes^{i j} \neg \varphi \quad \text { iff } \quad \exists y \in U^{j} .\left[\left(R^{i j}(x, y) \wedge y \models \varphi\right) \vee\left(\neg R^{i j}(x, y) \wedge y \models \neg \varphi\right)\right]
$$

\subsection{An illustrative instantiation}

As we mentioned earlier, first we will exemplify how the argument runs in order to make our formal reasoning easier to follow. For this reason, let us first take $n=3$ as a work-out case, before giving it for an arbitrary $n$. When necessary, for clarity in the notation we will write $\square^{n \cdot m}$ for $\square^{n m}$ —and similarly for $R^{n m}$.

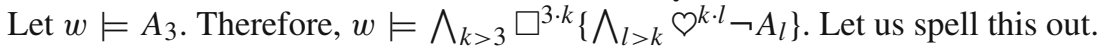

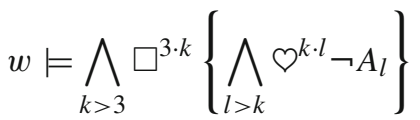

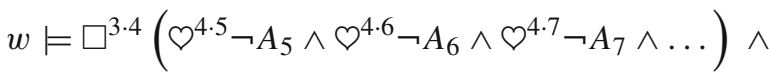

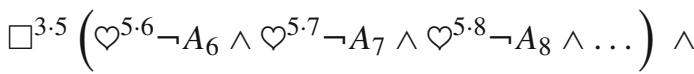

In particular, for example, $w \models \square^{3 \cdot 4} \Upsilon^{4 \cdot 7} \neg A_{7}$ and $w \models \square^{3 \cdot 5} \bigcirc^{5 \cdot 7} \neg A_{7}$. Therefore, it can be seen that for all $3<a<7, w \models \square^{3 \cdot a} \nabla^{a \cdot 7} \neg A_{7}$. Simply put, from agent 3 , through each other agent between 3 and 7 , it is possible to reach $\neg A_{7}$ via beliefassumption chain. We simply focused on player 7 and his assumption $A_{7}$, but the argument works for any player $n>4$ in our example.

The contradiction simply occurs when $A_{7}$ is hit by two different players in two different ways. In order to see it, consider $A_{5}$ (which can also reach $A_{7}$ by a beliefassumption chain). So, we have

$$
w \models \square^{3 \cdot 4} \bigcirc^{4 \cdot 5} \neg A_{5}
$$


where $\neg A_{5}$ is given as follows:

$$
\bigvee_{k>5} \diamond^{5 \cdot k}\left\{\bigvee_{l>k} \neg \nabla^{k \cdot l} \neg A_{l}\right\} .
$$

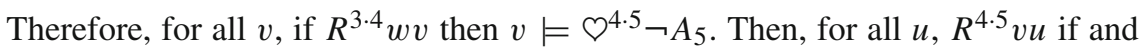
only if $u \models \neg A_{5}$. The use of the assumption modality here is crucial. It associates the set of states that falsifies $A_{5}$ with what is accessible from $v$ with $R^{4 \cdot 5}$.

Spelling this out, we have the following.

$$
\begin{aligned}
u \models & \diamond^{5.6}\left[\neg \oslash^{6.7} \neg A_{7} \vee \neg \oslash^{6.8} \neg A_{8} \vee \ldots\right] \vee \diamond^{5.7} \\
& \times\left[\neg \varnothing^{7.8} \neg A_{8} \vee \neg \oslash^{7.9} \neg A_{9} \vee \ldots\right] \vee \ldots
\end{aligned}
$$

The first disjunct in Sentence 2 (that is $\diamond^{5 \cdot 6}\left[\neg \nabla^{6 \cdot 7} \neg A_{7} \vee \ldots\right]$ ) suggests that there is a $t$ such that $R^{5 \cdot 6} u t$ and $t \models \neg \varnothing^{6 \cdot 7} \neg A_{7} \vee \neg \varnothing^{6 \cdot 8} \neg A_{8} \vee \ldots$

However, this is impossible. The first disjunct $\left(\neg \varnothing^{6 \cdot 7} \neg A_{7}\right)$ cannot be the case at $t$. Because it reduces to the following.

$$
\begin{aligned}
& t \models \neg \bigotimes^{6 \cdot 7} \neg A_{7}, \\
& \quad \text { iff } \quad \exists y \in U^{7}\left[\left(R^{6 \cdot 7}(x, y) \wedge y \models A_{7}\right) \vee\left(\neg R^{6 \cdot 7}(x, y) \wedge y \models \neg A_{7}\right)\right]
\end{aligned}
$$

But, this is impossible by our earlier observation: there is a state accessible via $R^{6 \cdot 7}$ that satisfies $\neg A_{7}$, and all the states accessible from $u$ satisfies $A_{7}$ due to the definition of the $\odot$ modality.

The argument can easily be extended to other disjunct in Statement 2 and their disjuncts. Thus, Statement 2 cannot have a consistent model. Therefore, each $A_{n}$ is false. As we observed earlier, then $A_{n}$ s are also true by definition. This is the paradox. ${ }^{5}$

\subsection{The proof}

Let us now go back to the formal argument. In what follows, we shall present a semantical approach to show how the set of sentences in Sentence 1 cannot have a standard model.

We first start with the following lemmata where $\equiv$ denotes the logical equivalence in the usual sense.

\section{Lemma 2.1}

1. $\nabla^{i j} \varphi \rightarrow \square^{i j} \varphi$

2. $\varnothing^{i j}(\varphi \wedge \psi) \equiv \varnothing^{i j} \varphi \wedge \varnothing^{i j} \psi$

\footnotetext{
5 As pointed out by one of the referees of this journal, it is important to notice the non-finitistic nature of this reasoning in this argument. We show that there is no classical model for the given sentence, not proving a contradiction-which would require non-finitistic resources obviously.
} 


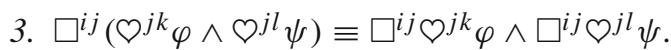

4. $\diamond^{i j}\left(\bigcirc^{j k} \varphi \vee \bigcirc^{j l} \psi\right) \equiv \diamond^{i j} \bigcirc^{j k} \varphi \vee \diamond^{i j} \bigcirc^{j l} \psi$

5. $\varnothing^{i j}\left(\square^{j k} \varphi \wedge \square^{j l} \psi\right) \equiv \varnothing^{i j} \square^{j k} \varphi \wedge{\bigcirc^{i j}}^{j l} \psi$

Proof The proofs follow immediately from the semantical definitions and the basic facts for the modal operators.

Lemma 2.2 If $w \models A_{n}$, then for all $p, q$ with $n<p<q ; w \models \square^{n p} \emptyset^{p q} \neg A_{q}$.

Proof The proof follows immediately from the semantical definitions of the modalities, $A_{n}$ and Lemma 2.1 .

Lemma 2.2 has some interesting consequences. For example, if $w \models A_{3}$, then we

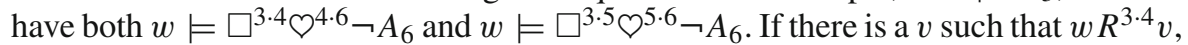
then $R^{4 \cdot 6}(v)=\left|\neg A_{6}\right|$. Moreover, if there is another $v^{\prime} \neq v$ such that $w R^{3 \cdot 4} v^{\prime}$, then $R^{4 \cdot 6}(v)=\left|\neg A_{6}\right|=R^{4 \cdot 6}\left(v^{\prime}\right)$. Similar argumentation shows that $R^{5 \cdot 6}(u)=\left|\neg A_{6}\right|=$ $R^{5 \cdot 6}\left(u^{\prime}\right)$ for some $u, u^{\prime}$ with $w R^{5 \cdot 6} u$. We can generalize this result as follows.

Theorem 2.3 If $w \models A_{n}$, then for all $p, p^{\prime}, q$ with $n<p<q$ and $n<p^{\prime}<q$; we have $R^{p q}(v)=R^{p^{\prime} q}\left(v^{\prime}\right)$ for all $v \in U^{p}$ and all $v^{\prime} \in U^{p^{\prime}}$.

Proof By Lemma 2.2, we observe that $w \models A_{n}$ implies both $w \models \square^{n p} \bigcirc p q \neg A_{q}$ and $w \models \square^{n p^{\prime}} \varnothing p^{\prime} q^{\prime} \neg A_{q}$ for $n<p<q$ and $n<p^{\prime}<q^{\prime}$.

Then, $\forall v \in U^{p}, w R^{n p} v$ implies that $v \models \rho^{p q} \neg A_{q}$. However, the last statement means that $\forall u .\left(v R^{p q} u \leftrightarrow u \models \neg A_{q}\right)$. Then, we observe that $\left|\neg A_{q}\right|=R^{p q}(v)$. Similarly, $\forall v^{\prime} \in U^{p^{\prime}}, w R^{n p^{\prime}} v$ implies that $v^{\prime} \models \rho^{p^{\prime}} q \neg A_{q}$ which yields that $R^{p q}(v)=$ $R^{p^{\prime} q}\left(v^{\prime}\right)$. The choices of $v$ and $v^{\prime}$ were arbitrary, thus the result follows.

Corollary 2.4 If $w \models A_{n}$, then $\square^{n p} \varnothing^{p q} \varphi \leftrightarrow \square^{n p^{\prime}} \wp^{p^{\prime} q} \varphi$ for $n<p<q$ and $n<p^{\prime}<q$.

Now, assume that $w \models A_{n}$ for some arbitrary $n$. Game theoretically, this means that player $n$ believes that all the other players after him assume that the assumptions of each player after them are false.

We can rewrite $w \models A_{n}$ as follows:

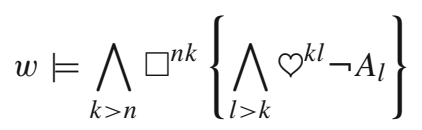

where $\neg A_{n}$ is given as follows:

$$
\neg A_{n}=\bigvee_{k>n} \triangleright^{n k}\left\{\bigvee_{l>k} \neg \oslash^{k l} \neg A_{l}\right\}
$$


Explicitly, we have the following for $w \models A_{n}$ :

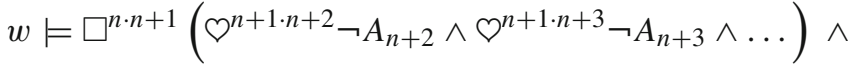

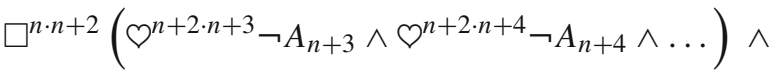

Now, similar to considering the Cantorian diagonal formula for the BK paradox, let us take the very first conjunct (after a quick application of Lemma 2.1) in Sentence 3:

$$
w \models \square^{n \cdot n+1} \bigcirc^{n+1 \cdot n+2} \neg A_{n+2}
$$

Spelling out $\neg A_{n+2}$, we obtain:

$$
\begin{aligned}
w \models \square^{n \cdot n+1} \bigcirc^{n+1 \cdot n+2} & \{ \\
& \diamond^{n+2 \cdot n+3}\left(\neg \bigcirc^{n+3 \cdot n+4} \neg A_{n+4} \vee \neg \bigotimes^{n+3 \cdot n+5} \neg A_{n+5} \vee \ldots\right) \vee \\
& \diamond^{n+2 \cdot n+4}\left(\neg \bigotimes^{n+4 \cdot n+5} \neg A_{n+5} \vee \neg \bigotimes^{n+4 \cdot n+6} \neg A_{n+6} \vee \ldots\right) \vee \\
& \vdots \\
& \}
\end{aligned}
$$

After arbitrarily many applications of Lemma 2.1, the above sentence implies the following.

$$
\begin{aligned}
w \models & \square^{n \cdot n+1} \bigcirc^{n+1 \cdot n+2} \triangleright^{n+2 \cdot n+3} \neg \bigcirc^{n+3 \cdot n+4} \neg A_{n+4} \vee \\
& \square^{n \cdot n+1} \bigcirc^{n+1 \cdot n+2} \triangleright^{n+2 \cdot n+3} \neg \bigcirc^{n+3 \cdot n+5} \neg A_{n+5} \vee \\
& \ldots \vee \\
& \square^{n \cdot n+1} \triangleright^{n+1 \cdot n+2} \triangleright^{n+2 \cdot n+4} \neg \bigcirc^{n+4 \cdot n+5} \neg A_{n+5} \vee \\
& \square^{n \cdot n+1} \bigcirc^{n+1 \cdot n+2} \triangleright^{n+2 \cdot n+4} \neg \bigcirc^{n+4 \cdot n+6} \neg A_{n+6} \vee \\
& \ldots \vee
\end{aligned}
$$

This is the first conjunct $\square^{n \cdot n+1} \bigcirc^{n+1 \cdot n+2} \neg A_{n+2}$ of $A_{n}$ that is assumed to be satisfied at $w$. 
Now, we will show that each and every disjunct in Sentence 4 is falsified. Let us start with the first disjunct. The following statement

$$
\square^{n \cdot n+1} \triangleright^{n+1 \cdot n+2} \triangleright^{n+2 \cdot n+3} \neg \bigotimes^{n+3 \cdot n+4} \neg A_{n+4}
$$

appearing in Sentence 4 means that

$$
\begin{aligned}
& \forall v \cdot \forall u \cdot \exists t . \\
& \quad\left(w R^{n \cdot n+1} v \rightarrow\left(v R^{n+1 \cdot n+2} u \leftrightarrow\left(u R^{n+2 \cdot n+3} t \wedge t \models \neg \bigotimes^{n+3 \cdot n+4} \neg A_{n+4}\right)\right)\right)
\end{aligned}
$$

But, by Theorem 2.3, this is impossible because for all $l$ such that $n<l<n+4$, $\square^{n \cdot l} \nabla^{l \cdot n+4} \neg A_{n+4}$. In particular, for $l=n+3$. Therefore, any state that can see a $U^{n+4}$-state contradicts the first disjunct in Sentence 4. Specifically, at $w$, the statement $\square^{n \cdot n+3} \bigcirc^{n+3 \cdot n+4} \neg A_{n+4}$ can be satisfied at a $t$ such that $t \models \mathrm{Q}^{n+3 \cdot n+4} \neg A_{n+4}$. If $t$ accessed from the intermediate state is unique, this is necessarily a contradiction. Witnesses contradicting the Sentence 5 can be multiplied.

This method easily generalizes to each and every disjunct in Sentence 4 and then to each and every conjunct in Sentence 3 . The choice of $n$ was arbitrary, thus each $A_{n}$ in Statement 1 fails.

So far, this simply shows that the configuration of beliefs and assumptions about players given in Statement 1 is false.

But, if each $A_{i}$ is false then each $\neg A_{i}$ is true. Then, $\Phi^{k l} \neg A_{l}$ for each $l>k$ is satisfiable in some model: simply add a state $x_{l}$ into $U^{l}$ which is accessible from some $U^{k}$ state such that $x_{l} \models A_{l}$. Thus, $\bigwedge_{l>k} \bigcirc^{k l} \neg A_{l}$ is satisfiable in some model. In a similar way, make every state in $U^{k}$ that can see a $U^{l}$ state accessible from $U^{1}$ for each $k>1$. This makes $\bigwedge_{k>1} \square^{1 \cdot k}\left\{\bigwedge_{l>k} \bigcirc^{k l} \neg A_{l}\right\}$ satisfiable. But, that is $A_{1}$ which we previously thought false. Contradiction.

Therefore, the configuration of beliefs and assumptions about players given in Statement 1 is semantically impossible-it cannot have a classical model.

This is a non-self-referential extension of the Brandenburger-Keisler paradox to $\omega$-many players in a Yabloesque fashion.

\subsection{Discussion}

The formal results we have presented so far have some immediate relevance to various major issues in logic and games, such as $\omega$-categoricity and infinitary games. Without any doubt, these issues are deep and complicated, and they have been argued for or against countless times within the context of Yablo's paradox. In what follows, we only consider the issues that are directly relevant to our current work, highlighting the fact that the YBK paradox suggests further insights for these issues. While doing so, we will also identify some open questions in the subject, promising future research directions. Furthermore, the following discussion gives it a context when we present a paraconsistent model for the paradox. 


\subsubsection{Assumption modality and the diagonal formula}

Assumption modality $\varnothing^{i j}$ is essential in the construction of the paradox. Without it, it is not possible to generate the YBK paradox. For example, the following set of sentences about players' beliefs is not inconsistent. ${ }^{6}$

$$
\begin{aligned}
A_{1}^{\prime} & :=\bigwedge_{k>1} \square^{1 k}\left\{\bigwedge_{l>k} \square^{k l} \neg A_{l}^{\prime}\right\} \\
A_{2}^{\prime} & :=\bigwedge_{k>2} \square^{2 k}\left\{\bigwedge_{l>k} \square^{k l} \neg A_{l}^{\prime}\right\} \\
A_{3}^{\prime} & :=\bigwedge_{k>3} \square^{3 k}\left\{\bigwedge_{l>k} \square^{k l} \neg A_{l}^{\prime}\right\} \\
\vdots &
\end{aligned}
$$

The belief modality in the above set of sentences generates distinct belief states which falsify the assumption without associating them with each other. In other words, for example, if $A_{4}^{\prime}$ is false at some state accessible by $\square^{2 \cdot 4}$ this does not associate it to the states that falsify the very formula but accessible by $\square^{3 \cdot 4}$. The reason for that is the weak semantics of the belief modality (compared to that of the assumption modality).

More formally, as Yablo also argued, what we would have in this case is a "downward facing tree with $\omega$ branches descending from each node" which satisfies the $A^{\prime}$ sentences, as illustrated below (Yablo 2004). We leave the details to the reader to construct such a tree model for Sentence 6.

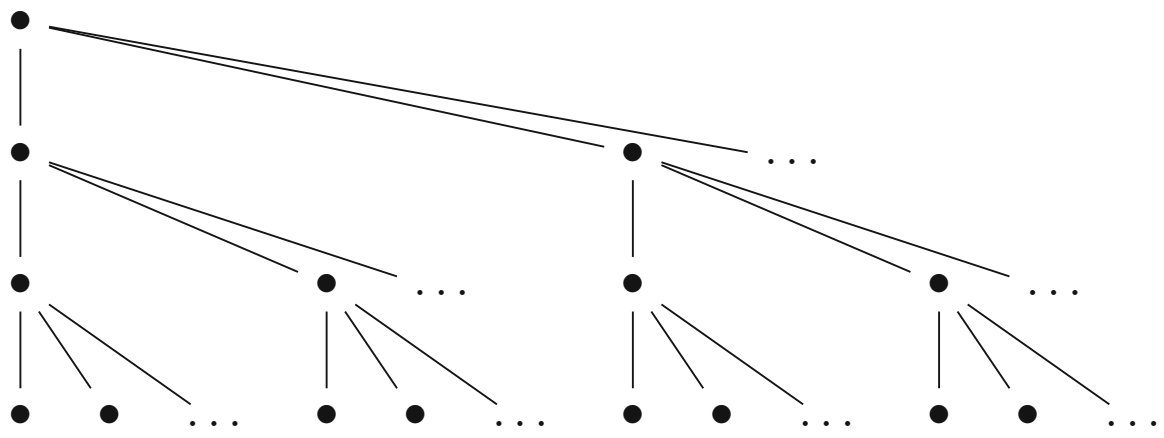

This case, however, is compatible with Sorensen's queue example (Sorensen 1998). Particularly, from Sorensen's queue sentence "Some of the students behind be are now thinking an untruth", it can be obtained the sentence "Some of the students behind me are now thinking about an untruth that the students behind them are thinking". As

$\overline{6}$ In order to prevent any possible confusion, we denote the following assumptions with $A_{n}^{\prime}$ instead of $A_{n}$. 
these thoughts and beliefs are not necessarily associated to each other in any way due to the lack of "assumptions", Sentence 6 can be viewed as a nested, infinitary Sorensen queue.

This remark is essential. Because it produces another unsatisfiable (not paradoxical) sentence akin to Cantor's sentence. Using nested queues, it is possible to construct a model where the Cantor sentence and Sentence 6 cannot simultaneously be satisfied. In an informal way, let us consider the set of sentences in Sentence 6 as follows where $F_{x \cdot y}$ denotes the assumptions (here $A^{\prime}$ s).

$$
\begin{aligned}
& \square^{1 \cdot 2} \square^{2 \cdot 3} F_{1 \cdot 2,2 \cdot 3} \wedge \square^{1 \cdot 2} \square^{2 \cdot 4} F_{1 \cdot 2,2 \cdot 4} \wedge \square^{1 \cdot 2} \square^{2 \cdot 5} F_{1 \cdot 2,2 \cdot 5} \wedge \ldots \\
& \square^{1 \cdot 3} \square^{3 \cdot 4} F_{1 \cdot 3,3 \cdot 4} \wedge \square^{1 \cdot 3} \square^{3 \cdot 5} F_{1 \cdot 3,3 \cdot 5} \wedge \square^{1 \cdot 3} \square^{3 \cdot 6} F_{1 \cdot 3,3 \cdot 6} \wedge \ldots \\
& \quad \vdots \\
& \square^{n \cdot k} \square^{k \cdot k+1} F_{n \cdot k, k \cdot k+1} \wedge \square^{n \cdot k} \square^{k \cdot k+2} F_{n \cdot k, k \cdot k+2} \wedge \square^{n \cdot k} \square^{k \cdot k+3} F_{n \cdot k, k \cdot k+3} \wedge \ldots
\end{aligned}
$$

If a model $M$ is constructed to satisfy Sentence 6 , we can generate a diagonal sentence $\delta$ such that $M \not \models \delta$. The sentence $\delta$ is defined by taking the diagonal of the above set of sentences following the Cantorian methodology. Therefore, $\delta$ is given as follows.

$$
\begin{aligned}
\delta:= & \neg \square^{1 \cdot 2} \square^{2 \cdot 3} F_{1 \cdot 2,2 \cdot 3} \wedge \neg \square^{1 \cdot 3} \square^{3 \cdot 5} F_{1 \cdot 3,3 \cdot 5} \\
& \wedge \cdots \wedge \neg \square^{n \cdot n+1} \square^{n+1 \cdot n+3} F_{n \cdot n+1, n+1 \cdot n+3} \wedge \ldots
\end{aligned}
$$

Notice that $\delta$ is indeed simultaneously unsatisfiable (classically) in a model $M$ satisfying the Sentence 7 . The first conjunct of $\delta$ refutes the first sentence, the second conjunct refutes the second sentence, and the $n$th conjunct in $\delta$ refutes the $n$th sentence in the Sentence 6.

This procedure is very common. Indeed, a similar approach for Yablo's paradox was also given by Cook (2014).

Finally, the following result sums up the discussion.

Theorem 2.5 Let $M$ be a belief model. Let $M \models A_{i}^{\prime}$ for each $i \in \omega$ where $A_{i}^{\prime}=$ $\bigwedge_{k>i} \square^{i \cdot k}\left\{\bigwedge_{l>k} \square^{k \cdot l} \neg A_{l}^{\prime}\right\}$. Then, there exists a sentence $\delta$ such that $M \forall \delta$.

The Sentence $\delta$ is essential to construct the Gödelian self-referentiality using the truth predicate in the standard way. The truth theoretical narration of this discussion falls outside the scope of this work, thus omitted.

Nevertheless, the above discussion immediately relates the YBK paradox to $\omega$ categoricity.

\subsubsection{Categoricity}

As argued by Ketland, the Yablo sentences are not satisfiable on the standard model of arithmetic, thus they are " $\omega$-inconsistent" (Ketland 2005). This observation suggests 
that the YBK paradox can be satisfied in a game model with $\omega+1$ players, a nonstandard model for the game. As every finite set of $A_{n} \mathrm{~s}$ in Sentence 1 are satisfiable, by compactness, there must exist a model for the YBK sentences.

For completeness of our arguments, let us first see how every finite set of $A_{n} \mathrm{~s}$ are satisfiable. In the original Yablo's paradox, this is an easy task. For a finite set of Yablo sentences $\left\{S_{f(1)}, \ldots, S_{f(n)}\right\}$ where $f$ is an strictly increasing injective indexing function, a model can be given as follows. As $f$ is strictly increasing, the sentence with the highest index is $S_{f(n)}$. Let $S_{f(n)}$ be true. Therefore, for each $k>f(n), S_{k}$ is untrue. Then, let all the other $S_{f(i)}$ for $i<n$ be false. As $f(i)<f(n)$ for $i<n, S_{f(i)}$ cannot hold for $i<n$ as $S_{f(n)}$ is true. In conclusion, in a finite set of Yablo sentences, simply let the sentence with the highest index be true, then the rest will be false. This is a model for a finite set of Yablo Sentences.

For the YBK sentences, we reason similarly. Given a finite set of assumptions $\left\{A_{f(1)}, \ldots, A_{f(n)}\right\}$ where $f$ is an strictly increasing injective indexing function, we force $A_{f(n)}$ be true and the rest false. Thus, every finite set of $A_{n} \mathrm{~s}$ are satisfiable, and as we argued, then there exists a non-standard model of the YBK paradox, which can be constructed similarly as in Ketland (2005). The details of this procedure is very similar to the Yablo case and left to the reader.

The significant contribution of Ketland's work is that it gives uniform schemes for Yablo's paradox (Ketland 2005). It is thus important to see if the YBK paradox fits in any of these schemes. The uniform homogeneous Yablo scheme which Ketland gives is as follows.

$$
\forall x[\varphi(x) \leftrightarrow \forall y(\psi(y, x) \rightarrow \neg \varphi(y))]
$$

Let us start with the following generalization to obtain the Uniform Homogeneous Yablo-Brandenburger-Keisler scheme.

$$
\forall x[\varphi(x) \leftrightarrow \forall y(\psi(y, x) \wedge \forall z(\xi(z, y) \rightarrow \neg \varphi(z)))]
$$

The above sentence is obtained by considering the additional relation (the assumption modality) as a precondition for the paradoxical result. Pursuing this line of generalization, we obtain the following uniform Yablo-Brandenburger-Keisler scheme for the YBK paradox.

$$
\forall x, y, z_{1}, z_{2}, \ldots\left[\varphi(x) \leftrightarrow\left(\psi(y, x) \wedge \bigwedge\left(\xi\left(z_{i}, y\right) \rightarrow \neg \varphi\left(z_{i}\right)\right)\right)\right]
$$

The above scheme indeed replaces the free variable $y$ in Ketland's version with a vector $\left(y, z_{1}, \ldots\right)$ with taking care of the additional predicate relations. The scheme in Sentence 8 raises the possibility of using different modal operators, as opposed to the assumption modality, to have a paradoxical situation similar to the YBK paradox. We leave inquiring about the possibility of this interesting theoretical direction to future work.

Similarly, the schemes presented above relate the discussion to curryesque iterations of the paradoxes. As Beall observed, a non-modal version of Yabloesque Curry paradox 
can be given (Beall 1999). Beall's version of the paradox can be summarized as follows for $n \geq 1$ :

$$
\lambda_{n}:=\text { If for all } \lambda_{i} \text { for } i>n \text { are true, then } 1=0 .
$$

It is not possible to obtain a curryesque YBK paradox directly from the scheme in Sentence 8 . By careful substitution, we obtain the following where $\lambda(i, n)$ denotes the formula $\lambda_{i}$ for all $i>n$.

$$
\forall n, i .\left[\lambda(n) \leftrightarrow\left(\lambda(i, n) \wedge \bigwedge_{i>n}(\lambda(i) \rightarrow \neg \lambda(i))\right)\right]
$$

The above formula reduces to the following as $\bigwedge_{i>n} \lambda(i)=\lambda(i, n)$.

$$
\forall n, i .[\lambda(n) \leftrightarrow(\lambda(i, n) \rightarrow \neg \lambda(i))]
$$

The above sentences still use the negation symbol and are not in the Curry form. Therefore, constructing a curryesque version of the YBK paradox is a natural next step for this project. In order to do justice to the subject, we leave it for future work.

The topic of $\omega$-consistency has been discussed not only from the view point of cardinality but also from the perspectives of first- and second-order logics and their proof theories. As observed by Barrio and Picollo in various occasions, Yablo's paradox behave differently in first- and second-order languages (Barrio 2010; Barrio and Picollo 2013; Picollo 2013). This difference stems from the compactness theorem and relates heavily to the categoricity results of truth theories and the failure of well-foundedness principle (Forster 2004). These observations constitute a significant discussion regarding whether $\omega$-categoricity should be required from truth theories, either in first- or second-order case. Moreover, it clarifies the differences between proof theoretical and semantical approaches to the mathematical analysis of paradoxes.

YBK paradox opens up a new direction for these discussions and relates the truth theoretical debate to a game theoretical framework. The relation between $\omega$ categoricity and game theoretical rationality, for example, is an unexplored and intriguing direction, which we leave for future work.

It is however an open question whether the first- or second-order formalizations of the YBK paradox would behave differently or whether the YBK sentence (or even the BK sentence) would not be paradoxical in second-order logic. The mathematical complexity of this research direction relies on van Benthem's theorem regarding the identification between bisimulation invariance of first-order logic and modal logic. Since a similar bisimulation invariance result is given between the second-order logic and modal fixed-point languages, a second-order reiteration of the YBK paradox necessarily requires a modal fixed-point language. In conclusion, the arguments we have presented so far demonstrates the logical breadth and depth of the YBK paradox and identifies some open questions in the field to stimulate future research. 


\subsubsection{Infinite games}

Mathematical approaches for omega-consistency focuses heavily on the formal aspects of the paradox. However, in this work the YBK paradox is formulated within the context of game theory. Therefore, the "game theoretical" aspects of the paradox raise some important issues for infinitary games.

Similar to Leitgeb's example, players possessing Yabloesque beliefs about other players are indeed infinitary: "[their] mental capacities are infinite in two respects: (1) they are able to bear in mind infinitely long linguistic items, and (2) they can hold infinitely may linguistic items before their minds" (Leitgeb 2005). Therefore, our games may be found unreasonable.

This is a valid criticism. Yet, this relates to even broader issues in logic and games, including epistemic omniscience (if an agent knows $\varphi$, he knows its deductive closure) and common knowledge (which has an infinitary definition). Briefly put, our approach is no exception to the problems of defining simple and foundational concepts in a logical framework.

Additionally, as it was underlined several times earlier, limitations of set theory (including the well-foundedness principle) affects the way the paradoxes are formalized, and game theoretical paradoxes are no exception (Heifetz 1996; Başkent 2015). Therefore, creating infinitary chains of beliefs and assumptions, with or without wellfoundedness, seems to be a problematic point as non-well-founded set theory do not seem to remedy self-referential paradoxes (Moss 2005).

Moreover, the YBK paradox enjoys a kind of exhaustiveness which is game theoretically not immediate to give an account for. As it is formulated in Sentence 1 and observed further in Lemma 2.2, the paradox forces each player to have beliefs about every other player after him in such a way that these players will have assumptions about all the players after them. This seems too much to ask for game theoretically. Or is that so?

On the contrary, it is relatively easy to find game theoretical examples discussing self-referentiality and/or non-well-foundedness. Another simple game theoretical example, similar to Sorensen's, for the YBK paradox might be a queue of people waiting to check-in to Hilbert's Hotel. The Hotel is thought of having infinitely many number of rooms, precisely $\omega$-many. Then, it can be imagined that the people anxiously waiting to check in to the hotel may have beliefs about the other people waiting behind them. Moreover, those beliefs can reasonably contain some assumptions about these people. For example, a player $i$ can believe that some other person $j>i$ behind him may assume that he himself will get the last room with a perfect view, so that $\forall k . k>j$ will have an assumption that their rooms will not have a perfect view. The YBK paradox then appears if their beliefs and assumptions are arranged in the way given in Sentence 1. Notice, however, this is very similar to Priest's claim about the self-referentiality of the Yablo paradox that is each person in the queue has the same thought (Priest 1997).

Infinitary games do not only appear within the context of logic. A more mathematically sophisticated paradoxical game is the hypergame paradox discussed by Zwicker (1987). Let us consider the paradox from Bernardi and D'Agostino (1996): 
Consider games between two players $A$ and $B$. We use the word 'game' in two different meanings, to denote both a game in general and any particular competition between $A$ and $B$. Given a game $G$, we will say 'a game of $G$ ', to denote a single competition which proceeds according to the rules of $G$. Call a game $G$ founded if every game of $G$ must terminate after finitely many moves, that is, following the rules, it is impossible for a game of $G$ to go on for ever, even if a priori there is no fixed bound on the lengths of games of $G$.

Now, define the hypergame as follows: player $A$ chooses a founded game $G$, then player $B$ makes the first move in $G$ and the game continues according to the rule of $G$. (Of course, there are simple winning strategies for $A$, but we are not concerned with them - in fact, we only specify legal moves, while the result of the game, who wins and who loses, has no importance.) Does this game have an end? Since $G$ is founded, the answer is obviously 'yes'. In other words, hypergame is a founded game. As a consequence, in a game of hypergame, player $A$ can choose the hypergame itself as a founded game, giving $B$ the right to choose the founded game. But, if $B$ decides to be as bizarre as $A$ and also chooses hypergame, and $A$ in turn repeats 'let's play hypergame', and so on, we get an unfounded game in which both players move according to the rules of a founded game.

Therefore, there are games and game theoretical situations involving infinitaryness and non-well-foundedness, and more importantly they appear quite naturally. The YBK paradox presents an interesting example regarding similar issues, with much more mathematical and logical sophistication.

The mathematical aspects of the YBK paradox are related to various other issues in logic, including fixed points. As we argued regarding the $\omega$-(in)consistency, Yablo's paradox (and perhaps also the YBK paradox) produce different results in first- and second-order cases (Picollo 2013). First- and second-order logics relate to modal paradoxes in a special way. The reason is that the bisimulation invariant fragment of first-order logic is indeed the classical modal logic, and the bisimulation invariant fragment of second-order logic is precisely the modal fixed-point logic. This immediately raises the possibility of representing the YBK paradox with modal-fixed points. Such extensions fall outside the scope of the current paper, thus left for future work.

Within this tradition, Bernardi's program investigates the mathematical connection between infinitary (that is non-founded games) and fixed-points (that is self-referentiality) in depth (Bernardi and D'Agostino 1996; Bernardi 2001). Such approaches give infinitariness a deeper conceptual meaning and relate it directly to non-well-foundedness in set theory. In fact, it is argued in Bernardi and D'Agostino (1996) that the method of diagonalization arises from self-referential paradoxes, as also argued in Yanofsky (2003). Nevertheless, this pattern is more familiar and much older than it looks. More generally, a single-page paper by Yuting gave essentially a similar paradox already in Yuting (1953). Yet, as mentioned in a footnote in Yuting (1953), the scheme of this paradox can be traced back to Quine's Mathematical Logic of 1951. What Yuting discussed was to give a paradox using non-well-founded sequences, which is, in fact, the basic idea behind most of such paradoxes. Indeed, such an approach was also given to analyze the BK paradox (Başkent 2015). 
Now, what is important for our purposes is the fact that both the BK paradox and the liar paradox can be expressed by fixed-points. In paraconsistent logic, which allows non-trivial inconsistencies, there are certain structures that can work with such fixedpoints and express inconsistencies in a sound way (Başkent 2015). In what follows, we consider topological spaces as a working case of such structures and construct a model that satisfies the YBK paradox, extending the use of topological models for paraconsistency from self-referential paradoxes to non-self-referential ones. This ties up the discussion and concludes the formal treatment of the YBK paradox by using a formal structure that has strong mathematical precision for various systems of logic and their semantics as well as for fixed-points.

\section{A paraconsistent model}

Our semantical treatment and the discussion which followed it establish the YBK paradox as a genuine and complex paradox. However, from a non-classical logical view point, this is not the end of the story. It is important to observe how an inconsistencytolerant system can model the paradox. Our formal approach therefore sheds lights on the debate whether Yablo's paradox (or the YBK paradox) is indeed self-referential. From a pragmatic perspective, our work complements an earlier attempt which suggested a paraconsistent model for the BK paradox (Başkent 2015). By applying the similar constructions to the YBK paradox, we verify the formal strength of paraconsistent game models which serves our general agenda of understanding paraconsistent games.

We will work with topological models which generate natural models for paraconsistent logics (Goodman 1981). What makes topological models stronger is that they are versatile. With some intuitive assumptions, they can give semantics for (classical) modal logics, intuitionistic logic and paraconsistent logic.

It is, however, important to note how our approach differs from Bernardi's (Bernardi $2009,2001)$. A natural approach to logical paradoxes is to study them by using fixedpoints. As Yablo-like paradoxes can be viewed as an infinite sequences of 0 s and $1 \mathrm{~s}$, this collection of sequences can be considered as a topological space. Thus, a topological investigation of truth-value sequences and their fixed-points relate the paradox directly to topological paradoxes.

Our line of research, however, is semantical. Topological spaces can also be viewed as the underlining structure for the extensions of logical formulas, which in turn create a paradox. Thus, replacing the formulas with their topological and set theoretical extensions promises a more structural analysis of the paradox and the role of logic in these paradoxes. This is the direction we are pursuing in this work.

Let us start with definitions. Given a non-empty set $S$, a collection of subsets $\sigma$ of $S$ is called a topology if it satisfies the following conditions:

- $S$ and $\emptyset$ are in $\sigma$,

- $\sigma$ is closed under arbitrary unions and finite intersections.

We call the tuple $(S, \sigma)$ a "topological space". The elements of $\sigma$ are called "opens" while their complements are "closeds". The interior operator Int(.) for a set $O$ returns the largest open set contained in $O$. Similarly, the closure operator $\mathrm{Clo}(\cdot)$ for a set $K$ 
returns the smallest closed set containing $K$. For a set $O$, the boundary of $O$ is defined as $\mathrm{Clo}(O)-\operatorname{Int}(O)$ and denoted as $\partial(O)$.

Topological spaces are central in various approaches to paraconsistent logics and their algebraic structures. For example, Lawvere discussed the role of boundary operator in co-Heyting algebras, which can be viewed as the algebraic structures of some paraconsistent logics (Lawvere 1991). From a topological perspective, the boundary operator $\partial(O)$ is essential to give semantics for paraconsistent logics in topological spaces (Goodman 1981; Mortensen 2000; Başkent 2013).

In the topological semantics for the classical modal logic, the operators Int and Clo are identified by $\square$ and $\diamond$ modalities as follows: $|\square \varphi|:=\operatorname{lnt}(|\varphi|)$. That is, the extension of a $\square \varphi$ is defined as the interior of the extension of $\varphi$. Similarly, we put $|\nabla \varphi|:=\operatorname{Clo}(|\varphi|)$. Therefore, in classical logic, modal operators necessarily generate open or closed sets. Nevertheless, ground formulas (formulas with no modalities appearing in them) do not necessarily generate opens or closeds sets in classical logic.

But it is possible to stipulate that the extensions of propositional variables to be closed sets. This stipulation works well with conjunction and disjunction as the finite intersection (and respectively, the union) of closed sets is closed. However, negation has to be handled carefully as the complement of a closed set is not necessarily closed, but open. Therefore, we define a special negation, a paraconsistent negation, $\sim$ as the closure of the complement. Then, what we obtain is a co-Heyting algebra (Başkent 2013, 2015).

Now we construct a paraconsistent model for the YBK paradox. For agent $i$, we take the corresponding non-empty type space $S_{i}$ and define topologies with closed sets $\sigma_{i}$. For example, for Player 3, the type space will be denoted by $S_{3}$ with a topology $\sigma_{3}$. In order to make this approach interactive, we define a function $s^{i j} \subseteq S_{i} \times S_{j}$ which associates states for player $i$ with the states of $j$. For example, for player $i$, at states from $S_{i}, s^{i j}$ returns a closed set $K \in \sigma_{j}$. We write $s^{i j}(w, K)$ means that at state $w \in S_{i}$, player $i$ believes that states $k \in K$ are possible for $j$.

The model is a tuple $\left(\left\{S_{i}\right\}_{i \in I},\left\{\sigma_{i}\right\}_{i \in I},\left\{s^{i j}\right\}_{i, j \in I}, V\right)$ where $V$ is a valuation defined in the standard way. The syntax for this system is similar to what we have given in Sect. 2.2, and restated here with the paraconsistent negation symbol $\sim$ which we discussed above and for $p \in \mathbf{P}$ where $i \neq j \in I$ :

$$
\varphi:=p|\sim \varphi| \varphi \wedge \varphi\left|\square^{i j} \varphi\right| \oslash^{i j} \varphi
$$

The dual modalities are defined as usual with the paraconsistent negation.

The paraconsistent topological semantics for this language is given as follows for negation and the modal operators as the Booleans are standard. For a set $X$, the complement of $X$ will be denoted by $X^{c}$.

$$
\begin{aligned}
& |\sim \varphi|=\operatorname{Clo}\left(K^{c}\right) \\
& \left|\square^{i j} \varphi\right|=\left\{w \in S_{i}: \exists K \in \sigma_{j} \text { with } s^{i j}(w, K) \text { and } K \subseteq|\varphi|\right\} \\
& \left|\varnothing^{i j} \varphi\right|=\left\{w \in S_{i}: \exists K \in \sigma_{j} \text { with } s^{i j}(w, K) \text { and } K=|\varphi|\right\}
\end{aligned}
$$


For easy read, we give the semantics for the modalities in the traditional sense as follows.

$$
\begin{array}{lll}
w \models \square^{i j} \varphi & \text { iff } & \exists K \in \sigma_{j} \text { with } s^{i j}(w, K) \rightarrow \forall v \in K . v \models \varphi \\
w \models \nabla^{i j} \varphi & \text { iff } & \forall K \in \sigma_{j} \text { with } s^{i j}(w, K), \exists v \in K \text { such that } v \models \varphi \\
w \models \nabla^{i j} \varphi & \text { iff } & \exists K \in \sigma_{j} \text { with } s^{i j}(w, K) \leftrightarrow \forall v \in K . v \models \varphi
\end{array}
$$

Now we construct a topological paraconsistent models for the YBK paradox. Let us reconsider the set of modal formulas given in Sentence 1.

$$
\begin{aligned}
& A_{1}:=\bigwedge_{k>1} \square^{1 k}\left\{\bigwedge_{l>k} \nabla^{k l} \neg A_{l}\right\} \\
& A_{2}:=\bigwedge_{k>2} \square^{2 k}\left\{\bigwedge_{l>k} \nabla^{k l} \neg A_{l}\right\} \\
& A_{3}:=\bigwedge_{k>3} \square^{3 k}\left\{\bigwedge_{l>k} \nabla^{k l} \neg A_{l}\right\}
\end{aligned}
$$

We will construct a model and a possible world $w$ in it that satisfy the above set of formulas step by step starting with player 1 and $A_{1}$. Now, for player 1 , take $w_{1} \in S_{1}$ and consider $A_{1}$. For each $k>1$, construct $K_{1 k} \in \sigma_{k}$ such that $s^{1 k}\left(w_{1}, K_{1 k}\right)$ and each $v_{k} \in K_{1 k}$ satisfies $\bigwedge_{l>k} \bigcirc^{k l} \sim A_{l}$. Therefore, for each $l>k$, there exists $U_{k l}$ that $s^{k l}\left(v_{k}, U_{k l}\right)$ such that every $u_{l} \in U_{k l}$ if and only if $u_{l} \in\left|\sim A_{l}\right|$. Let us unravel $\sim A_{l}$ as follows: $\sim A_{l}=\bigvee_{p>l} \diamond^{l p}\left\{\bigvee_{q>p} \sim \varnothing^{p q} \sim A_{q}\right\}$. This is a disjunctive statement. As our goal is to construct a counter-model, we will try to satisfy only one of the disjuncts and nested-disjuncts.

Now at $u_{l}$, include $\left(u_{l}, K_{1 p}\right)$ in $s^{l p}$ for all $p>l$. Thus, we have $s^{l p}\left(u_{l}, K_{1 p}\right)$. By construction of $A_{1}$ (hence of each $A_{i}$ ), each $v_{p} \in K_{1 p}$ satisfies $\bigwedge_{q>p} \rho^{p q} \sim A_{q}$, hence $\wp^{p q} \sim A_{q}$ for each $q>p$. Similarly, include $\left(v_{p}, x_{q}\right)$ for each $x_{q} \in \partial\left(\left|A_{q}\right|\right)$ into $s^{p q}$ in a way that $s^{p q}\left(v_{p}, \partial\left(\left|\sim A_{q}\right|\right)\right)$. Thus, $v_{p}=\sim \varnothing^{p q} \sim A_{q} \wedge \varnothing^{p q} \sim A_{q}$. We constructed a model in which we have $w_{1} \models A_{1}$.

This methodology can be extended inductively for each assumption $A_{i}$ which in turn builds the counter-model that satisfy each and every formula in Sentence 1. We skip the technical details of this straight-forward generalization process. The crucial observation is that the extension of a $\bigcirc$-formula uniquely identifies with the extension of the formula in question. However, some of the points in that extension may also satisfy the negation of the formula in question in paraconsistent models. This makes it quite straight-forward to construct the counter-model.

Bernardi defines unfounded chains for a sequence $\left(x_{n}\right)_{n \in \omega}$ and a function $f$, if $f x_{n+1}=f x_{n}$ for every $n$ (Bernardi 2001). The curious task of studying unfounded chains in paraconsistent topologies within epistemic game theoretical framework is left for future work.

So far, what we have achieved is to set up an adequate model in which YBK sentence can be satisfied. This point supports both the paraconsistent and modal agendas by 
suggesting an inconsistency-friendly system in game theory and a direct application of modal logic of games, respectively.

\section{Conclusion}

Paradoxes of game theory are intriguing both for philosophical logic and epistemic game theory. The current work relates closely to philosophical logic by extending the discussion on interactive paradoxes and the theory of truth, and to epistemic game theory by presenting an interesting epistemic game theoretical paradox. However, it is perfectly possible to view the YBK paradox as a modal logical argument, stripping it from its epistemic game theoretical content. Therefore, our presentation can very well be considered as a modal extension of Yablo's paradox.

It is important to note that the formal discussion we have presented here is illuminating for seeing whether Yablo's paradox is indeed self-referential or not, echoing Priest's arguments. The version we developed does not look self-referential at first glance, akin to the original paradox, yet Priest's arguments regarding the self-referential nature of the paradox somehow crystallizes here when we develop the counter-models.

The way we developed the game theoretical reading of Yablo's paradox by no means suggest that our method is the only way to obtain a multi-player iteration of Yablo's paradox or the only way to obtain a non-self-referential variation of the BK paradox. The plurality of such possibilities already point out a wide variety of future work possibilities which may shed some light on the algebraic or structural analysis of non-self-referential paradoxes.

An interesting direction to pursue along these lines is developing a Curryesque epistemic game theoretical paradox in which negation and falsity predicates are not used. The relation between the Curry and Yablo paradoxes are studied heavily (for instance, see Cook (2009)), therefore it remains to study its impact and applications of Yablurry paradoxes (as Cook calls them in Cook (2009)) in games.

Acknowledgements I am grateful to Leon Horsten for directing me to categoricity results. I acknowledge the constructive comments of the referees which helped improve the paper.

Open Access This article is distributed under the terms of the Creative Commons Attribution 4.0 International License (http://creativecommons.org/licenses/by/4.0/), which permits unrestricted use, distribution, and reproduction in any medium, provided you give appropriate credit to the original author(s) and the source, provide a link to the Creative Commons license, and indicate if changes were made.

\section{References}

Abramsky, S., \& Zvesper, J. (2015). From lawvere to Brandenburger-Keisler: Interactive forms of diagonalization and self-reference. Journal of Computer and System Sciences, 81(5), 799-812.

Barrio, E. A. (2010). Theories of truth without standard models and Yablo's sequences. Studia Logica, 96(3), 375-391.

Barrio, E. A., \& Picollo, L. M. (2013). Notes on $w$-inconsistent theories of truth in second-order languages. Review of Symbolic Logic, 6(4), 733-741.

Başkent, C. (2013). Some topological properties of paraconsistent models. Synthese, 190(18), 4023-4040.

Başkent, C. (2015). Some non-classical approaches to the Brandenburger-Keisler paradox. Logic Journal of the IGPL, 23(4), 533-552. 
Beall, J. C. (1999). Completing sorensen's menu: A non-modal yabloesque curry. Mind, 108(431), 737-739.

Beall, J. C. (2001). Is Yablo's paradox non-circular. Analysis, 61(3), 176-187.

Bernardi, C. (2001). Fixed points and unfounded chains. Annals of Pure and Applied Logic, 109(3), $163-178$.

Bernardi, C. (2009). A topological approach to Yablo's paradox. Notre Dame Journal of Formal Logic, 50(3), 331-338.

Bernardi, C., \& D’Agostino, G. (1996). Translating the hypergame paradox: Remarks on the set of founded elements of a relation. Journal of Philosophical Logic, 25(5), 545-557.

Brandenburger, A., \& Keisler, H. J. (2006). An impossibility theorem on beliefs in games. Studia Logica, $84,211-240$

Cook, R. T. (2009). Curry, Yablo and duality. Analysis, 69(4), 612-620.

Cook, R. T. (2014). The Yablo paradox. Oxford: Oxford University Press.

Forster, T. (2004). The significance of Yablo's paradox without self-reference. Logique et Analyse, 47(1858), 461-462.

Goldstein, L. (1994). A Yabloesque paradox in set theory. Analysis, 54(4), 223-227.

Goldstein, L. (2006). Fibonacci, Yablo and the cassationist approach to paradox. Mind, 115(460), 867-890.

Goodman, N. D. (1981). The logic of contradiction. Zeitschrift für Mathematische Logik und Grundlagen der Mathematik, 27(8-10), 119-126.

Hardy, J. (1995). Is Yablo's paradox liar-like? Analysis, 55(3), 197-198.

Heifetz, A. (1996). Non-well-founded type spaces. Games and Economic Behavior, 16, $202-217$.

Ketland, J. (2005). Yablo's paradox and $\omega$-inconsistency. Synthese, 145(3), 295-302.

Lawvere, F. W. (1991). Intrinsic co-heyting boundaries and the leibniz rule in certain toposes. In A. Carboni, M. Pedicchio, \& G. Rosolini (Eds.), Category theory (Vol. 1488, pp. 279-281)., Lecture Notes in Mathematics Berlin: Springer.

Leitgeb, H. (2005). Paradox by (non-well-founded) definition. Analysis, 65(4), 275-278.

Mortensen, C. (2000). Topological seperation principles and logical theories. Synthese, 125(1-2), 169-178.

Moss, L. S. (2005). Non-wellfounded set theory. In Zalta, E. N. (ed.), The Stanford Encyclopedia of Philosophy. Fall 2009 edition. http://plato.stanford.edu/archives/fall2009/entries/nonwellfounded-settheory.

Pacuit, E. (2007). Understanding the Brandenburger-Keisler belief paradox. Studia Logica, 86(3), 435-454.

Picollo, L. M. (2013). Yablo's paradox in second-order languages: Consistency and unsatisfiability. Studia Logica, 101(3), 601-617.

Priest, G. (1997). Yablo's paradox. Analysis, 57(4), 236-242.

Sorensen, R. A. (1998). Yablo's paradox and kindred infinite liars. Mind, 107(425), 137-155.

Yablo, S. (1985). Truth and reflection. Journal of Philosophical Logic, 14(3), 297-349.

Yablo, S. (1993). Paradox without self-reference. Analysis, 53(4), 251-252.

Yablo, S. (2004). Circularity and paradox. In T. Bolander, V. F. Hendricks, \& S. A. Pedersen (Eds.), Selfreference (pp. 165-183). Stanford: CSLI Publications.

Yanofsky, N. S. (2003). A universal approach to self-referential paradoxes, incompleteness and fixed points. The Bulletin of Symbolic Logic, 9(3), 362-386.

Yuting, S. (1953). Paradox of the class of all grounded classes. The Journal of Symbolic Logic, 18(2), 114.

Zwicker, W. S. (1987). Playing games with games: The hypergame paradox. The American Mathematical Monthly, 94(6):507-514. June-July 1987. 